\title{
Demora en el tratamiento quirúrgico del cáncer de pulmón
}

Torres García AJ. Demora en el tratamiento quirúrgico del cáncer de pulmón. An Med Interna (Madrid) 2005; 22: $359-360$.

El cáncer de pulmón es el cáncer más común tanto en términos de incidencia (1,04 millones de nuevos casos por año; $12,8 \%$ del total mundial de cánceres) como de mortalidad (921.000 muertes al año; 17,8\% del total mundial) (1). Esta incidencia y mortalidad están aumentando rápidamente, especialmente en los países en vías de desarrollo.

Por otra parte el cáncer de pulmón es una enfermedad de, globalmente considerada, muy mal pronóstico. La supervivencia a los cinco años en EE.UU. es del 14\% según estimaciones del "Surveillance, Epidemiology and End Results Programme”, y en Europa del $8 \%$. No obstante, existen diferencias entre los distintos países (2).

Una de las principales razones que inciden en este mal pronóstico es lo avanzado que se encuentran la mayor parte de los tumores cuando se diagnostican. En este sentido, el $78 \%$ de los pacientes con cáncer de pulmón tienen una enfermedad localmente avanzada o metastásica (estadios III y IV) cuando son diagnosticados, mientras que sólo el $22 \%$ se presenta con una enfermedad posiblemente curable (estadios I y II). Actualmente, la supervivencia media a los 5 años para los cánceres en estadios precoces (estadios I y II) esta comprendida entre el 40-80\% (70-80\% para el estadio I). Para estadios más avanzados (III y IV), la supervivencia a cinco años es fuertemente dependiente de la presencia o ausencia de afectación ganglionar ( $20 \%$ de media) o de la presencia de metástasis (casi $0 \%$ de supervivencia a los cinco años) (2).

Cuando hablamos de retraso o demora en el tratamiento de una determinada enfermedad, hemos de distinguir entre retraso en la presentación (intervalo existente entre la aparición del primer síntoma y el inicio de los exámenes diagnósticos); retraso diagnóstico (hasta que el diagnóstico es alcanzado) y retraso terapéutico (hasta que se inicia el tratamiento). En general se considera que, en lo que se refiere al carcinoma broncogénico que requiere cirugía, la suma de los retrasos diagnóstico y terapéutico no debería exceder 6-8 semanas (4256 días) (3).

En el estudio llevado a cabo en el área hospitalaria Virgen Macarena de Sevilla (4), es analizado el retraso terapéutico encontrado en un grupo de 46 pacientes intervenidos quirúrgicamente durante los años 2003 y 2004. La mediana de días de espera alcanzada fue de 31 días, con una media de 46,16 días y una desviación típica de 34,51. En un estudio similar en los objetivos planteados, pero con una muestra prospectiva y más amplia llevado a cabo por González Aragoneses y cols. del Grupo Cooperativo del Carcinoma Broncogénico de la Sociedad Española de Neumología y Cirugía Torácica (GCCBSEPAR) (5), la media de retraso terapéutico fue de 35 días (1154). En este estudio se investigó la influencia de este retraso terapéutico en la supervivencia de los pacientes intervenidos quirúrgicamente. Para ello, este parámetro, el retraso terapéutico fue incluido en un análisis multivariante tanto como variable cuantitativa como cualitativa, realizando una comparación entre diferentes intervalos de demora (1-20 vs. 21-40 vs. 41-60 vs. > 60 días). Los resultados del análisis multivariante mostraron que las únicas variables que influyeron en la supervivencia fueron la edad mayor de 70 años, los estadios clínicos I vs. II, y la estadificación patológica. Ni el tipo histológico ni la demora terapéutica (en sus diferentes intervalos) influyeron en la supervivencia de los pacientes y por ende en su pronóstico.

Es claro que uno de los problemas éticos y estratégicos de cualquier Sistema Sanitario (público o privado) es la existencia de importantes retrasos (de presentación, diagnósticos y terapéuticos) en la atención de sus usuarios. Esto es especialmente relevante y constituye una gran alarma social en el caso de los tumores malignos, y especialmente en el cáncer de pulmón. Ahora bien, aunque pueda inicialmente parecer extraño y contradictorio, no está universalmente demostrado y aceptado que estos retrasos impliquen un peor pronóstico para los pacientes con neoplasias malignas. Algunos estudios recientes acerca del carcinoma broncogénico (6) así como del cáncer de mama (7) han concluido que el retraso entre la aparición del primer síntoma o cuando el paciente es examinado por primera vez, y el tiempo de la cirugía influye en el sentido de que el tumor se diagnostica en un estadio más avanzado, lo cual consecuentemente implicaría un peor pronóstico.

Otros estudios (8), sin embargo, han demostrado, en tumores de diferente localización (pulmón, mama, estómago, colon y recto) y utilizando modelos de riesgos proporcionales de Cox, que el retraso diagnóstico no es un factor significativo 
para predecir la supervivencia. Aún más, en ciertos casos, como el cáncer de colon, un mayor retraso diagnóstico puede implicar un mejor pronóstico, reflejando que el resultado de la interacción tumor (con su propio comportamiento biológico)paciente puede arrojar un balance mucho más positivo que el que en teoría podría esperarse.

Por otra parte, el problema más importante para alcanzar una respuesta definitiva a esta cuestión es la imposibilidad de alcanzar suficiente evidencia científica mediante el diseño y desarrollo de estudios prospectivos y randomizados. Las razones éticas son obvias.

No obstante, a pesar de las dudas expuestas previamente acerca de la influencia o no de los "retrasos" o "demoras" en el pronóstico de los pacientes con carcinoma broncogénico que van a ser sometidos a tratamiento quirúrgico, es claro que retrasos terapéuticos prolongados ( $>45$ días) no son ética ni socialmente aceptables. Es preciso que Unidades o Grupos de Trabajo específicos profundicen en la optimización de la eficacia de los recursos implicados en estos procesos $(9,10)$.

Por otra parte, aunque el pronóstico del carcinoma broncogénico ha mejorado algo a lo largo de los últimos años (19602000), esto ha sido debido a la migración de estadios y a un mayor refinamiento y optimización de los tratamientos quirúrgico y quimioterápico. En contraste, la mortalidad debida a otros tipos de cánceres (excepto el de pulmón) ha disminuido en torno a un 25\% en el periodo 1950-1999 en Estados Unidos de Norteamérica. Esto se debe a una mejoría importante en los programas de "screening" o "cribaje" y de diagnóstico precoz (11). Asimismo, en el Reino Unido, la mortalidad por cáncer de mama en la mujer, cáncer de colon, recto, vejiga y melanoma también ha disminuido en los últimos cinco años (12).

Aunque hay estrategias de "screening" aceptadas para el cáncer de mama y el cáncer de cuello uterino, y recomendaciones para el cáncer de colon y de próstata en algunos países, no existe ninguna estrategia de salud pública diseñada para la detección precoz del cáncer de pulmón. Los estudios llevados a cabo en EE.UU. en los años 1980 (Clínica Mayo, Memorial Sloan Catering Cancer Center y Johns Hopkins) solo concluyeron que en los grupos sometidos a cribaje los individuos diagnosticados de cáncer de pulmón lo eran en estadios iniciales (estadios I y II) en una proporción mayor que en la población no sometida a estos estudios. Estos estudios realizados en varones fumadores no mostraron una reducción en la mortalidad por carcinoma broncogénico y podrían haber incurrido en varios sesgos metodológicos como el de sobrediagnóstico de enfermedad.

En los últimos dos años existe un interés progresivo por el relanzamiento de las estrategias de detección precoz del carcinoma broncogénico. En la conferencia internacional desarrollada en Italia en 1998 (13) se consensuó la necesidad de profundizar en estrategias de prevención primaria (no fumar) así como favorecer la financiación de estudios que analicen las posibilidades de las nuevas tecnologías (CT espiral o helicoidal, automatización de la citología de esputo, estudios de biología molecular, endoscopia fluorescente, etc.) para la detección precoz del carcinoma broncogénico.

En este sentido, los programas de deshabituación tabáquica sólo tienen unos porcentajes de éxito en torno al 10-20\%. Además, los individuos que han dejado de fumar constituyen un importante porcentaje de los casos de cáncer de pulmón diagnosticados de nuevo (casi un 50\% en la actualidad). Por lo tanto, los beneficios de los programas de cesación del hábito tabáquico son limitados en lo que se refiere a mejorar el pronóstico de los pacientes con cáncer de pulmón.

De ahí que, hoy en día, hemos de lanzar una llamada de atención clara e inequívoca para que se favorezcan los diseños de políticas de "screening" o cribaje que permitan alcanzar un diagnóstico lo más precoz posible del cáncer de pulmón. En este contexto, el control de la demora del tratamiento quirúrgico a implementar en estos pacientes deberá ser objetivo prioritario de todos los grupos de trabajos implicados en la atención de estos pacientes.

\section{A. J. TORRES GARCÍA}

\section{Servicio de Cirugía. Hospital Universitario San Carlos. Madrid}

\section{Bibliografía}

1. Landis SH, Murria T, Bolden S, Wingo PA. Cancer statistics 1999. CA Cancer J Clin 1999; 49: 44-6.

2. van Klaveren RJ, Habbema JDF, Pedersen JH, de Koning HJ, Oudkerk $\mathrm{M}$, Hoogsteden HC. Lung cancer screening by low-dose spiral computed tomography. Eur Respir J 2001; 18: 857-66.

3. Dische S, Gibson D, Parmar M, Saunders MI. Time course from first symptom to treatment in patients with non-small cell lung cancer referred for radiotherapy: a report by the CHART Steering Committee. Thorax 1996; 51: 262-5.

4. Almadana Pacheco V, Vázquez Martín M, Sánchez Varilla JM, Torres Gómez J, Dante García Ibarra H, Martínez Fuentes V. Demora desde el diagnóstico hasta el tratamiento quirúrgico de pulmón en el área hospitalaria Virgen Macarena de Sevilla. An Med Interna (Madrid) 2005; 22: 361-363.

5. González Aragoneses F, Moreno N, León P, Montan EG, Folque E, The Bronchogenic Carcinoma Cooperative Group of the Spanish Society of Pneumology and Thoracic Surgery (GCCB-S). Influence of delays on survival in the surgical treatment of bronchogenic carcinoma. Lung Cancer 2002; 36: 59-63.

6. Christensen ED, Harvald T, Jendresen M, Aggestrup S, Petterson G. The impact of delayed diagnosis of lung cancer on the stage at the time of operation. Eur J Cardiothorac Surg 1997; 12: 880-4.

7. Richards MA, Westcombe AM, Love SB, Littlejohns P, Ramírez AJ. Influence of delay on survival in patients with breast cancer: a systematic review. Lancet 1999; 353: 1119-26.

8. Porta M, Gallen M, Malats N, Planas J. Influence of "diagnostic delay" upon cancer survival: an analysis of five tumour sites. J Epidemiol Commun Health 1991; 45: 325-30.

9. Grupo Cooperativo de Carcinoma Broncogénico de SEPAR (GCCB-S), López Encuentra A, Martin de Nicolás JL, Casado López M, de Miguel Poch E, Marrón Fernández C. Demoras en el diagnóstico y en el tratamiento quirúrgico del carcinoma broncogénico. Arch Bronconeumol 1998; 34: 123-6.

10. Billing JS, Wells FC. Delays in the diagnosis and surgical treatment of lung cancer. Thorax 1996; 51: 903-6.

11. Rodu b, Cole P. The fifty-year decline of cancer in America. J Clin Oncol 2001; 19: 239-41.

12. Richards MA, Stockton D, Babb P, Coleman MP. Hiw many deaths have been avoided through improvements in cancer survival? BMJ 2000; 320: 895-8.

13. International Conference on prevention and Early Diagnosis of Lung Cancer. Varese, Italy. December, 9-10, 1998. 
$\mathbf{r}$

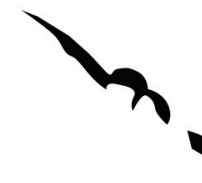

Staphylocodeus auheus

htaphy poccus epidenidis

lyeptoch ecus Anewnoḱa

4dnekoban er batumannd riegsiella opeunitiae Escherichie 501 Entelococoub faeckits Enterpoocoushaechym Bacterpides fragillis Legiondlla pneumophda i

¿DE AMPLIO ESPECTRO O MULTIRRESISTENCIA? 


\section{MNMV}

1.3.

Sociedad Andaluza de Patolozia Digestiva

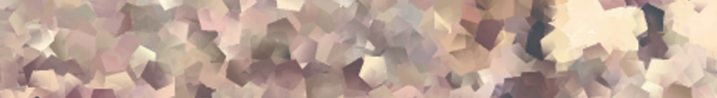

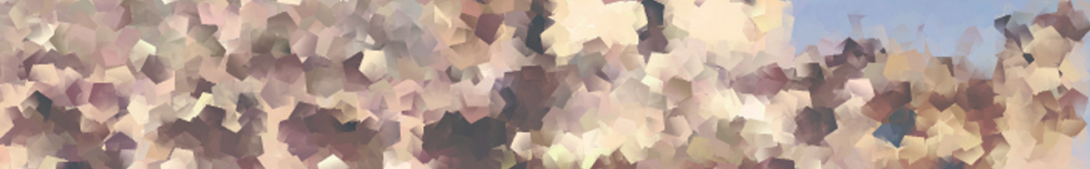

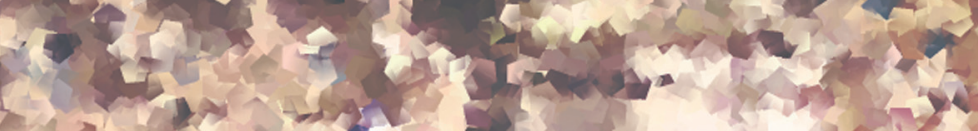

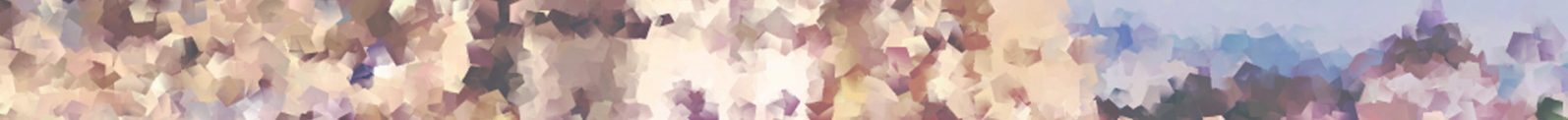

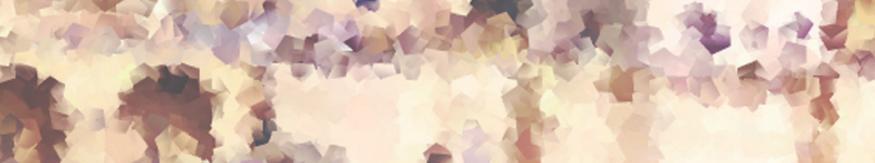

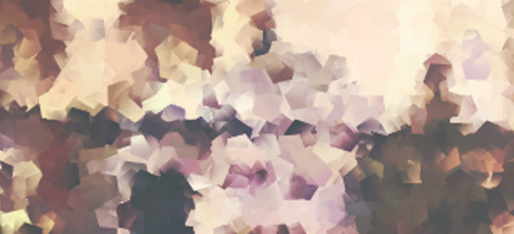

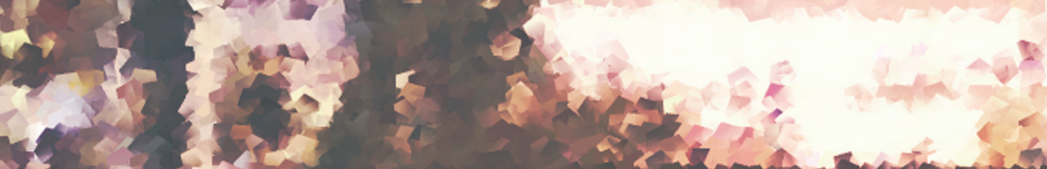
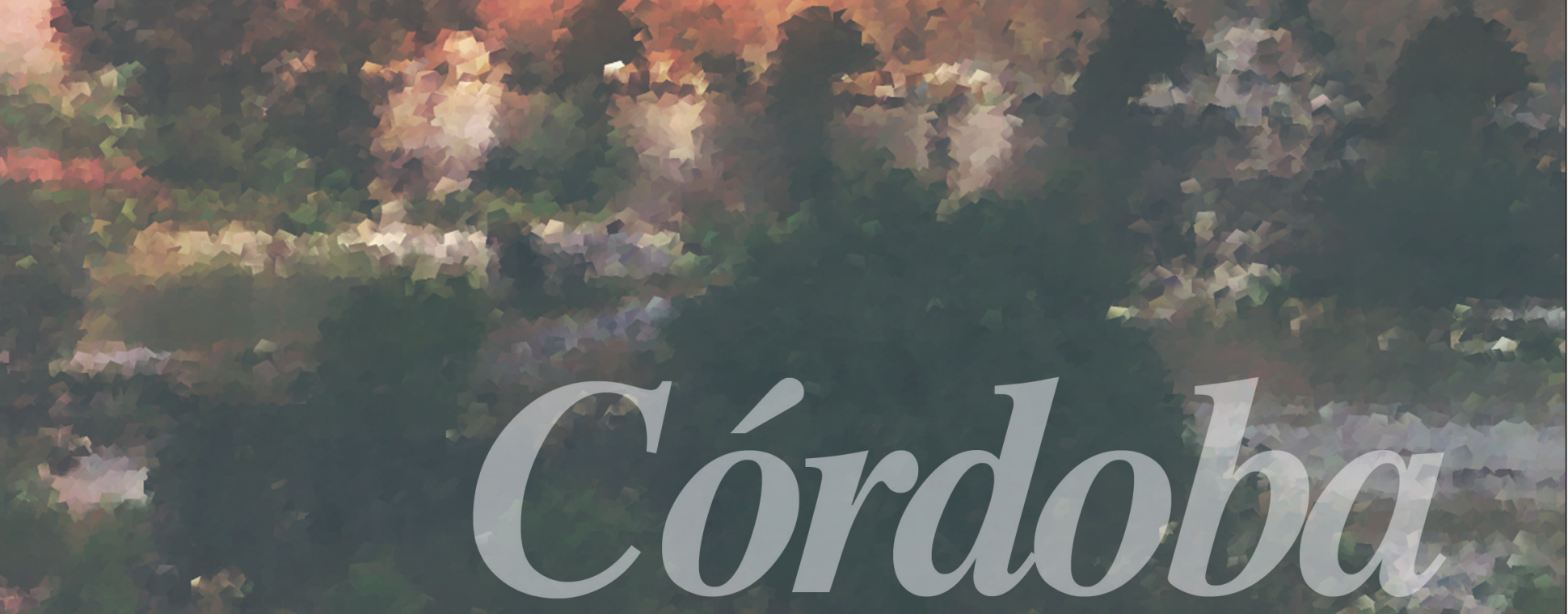

20,21 y 22 octubre de 2005

in

है

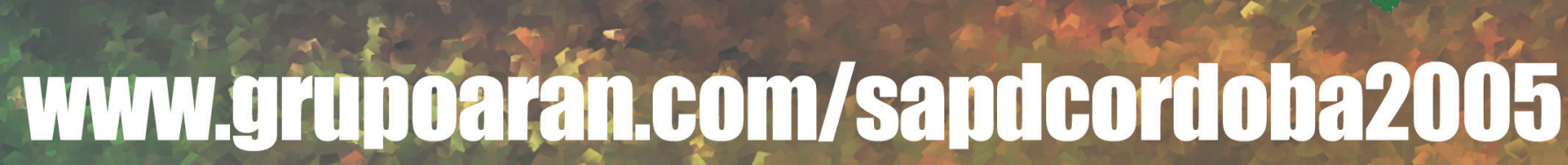

